\title{
Pleiotropy constrains the evolution of protein but not regulatory sequences in a transcription regulatory network influencing complex social behaviors
}

\author{
Daria Molodtsova ${ }^{1}$, Brock A. Harpur ${ }^{1}$, Clement F. Kent ${ }^{1+}$, Kajendra Seevananthan ${ }^{2}$ and Amro Zayed ${ }^{1}$ * \\ ${ }^{1}$ Department of Biology, York University, Toronto, ON, Canada \\ ${ }^{2}$ Department of Computer Science and Engineering, York University, Toronto, ON, Canada
}

Edited by:

Greg J. Hunt, Purdue University,

USA

\section{Reviewed by:}

Brian R. Johnson, University of

California, Davis, USA

Sandra M. Rehan, University of New

Hampshire, USA

\section{${ }^{*}$ Correspondence:}

Amro Zayed, Department of Biology, York University, 4700 Keele Street,

Toronto, ON M3J 1P3, Canada

e-mail: zayed@yorku.ca

${ }^{\dagger}$ Present address:

Clement F. Kent, HHMI Janelia Farm

Research Campus, Ashburn, VA,

USA
It is increasingly apparent that genes and networks that influence complex behavior are evolutionary conserved, which is paradoxical considering that behavior is labile over evolutionary timescales. How does adaptive change in behavior arise if behavior is controlled by conserved, pleiotropic, and likely evolutionary constrained genes? Pleiotropy and connectedness are known to constrain the general rate of protein evolution, prompting some to suggest that the evolution of complex traits, including behavior, is fuelled by regulatory sequence evolution. However, we seldom have data on the strength of selection on mutations in coding and regulatory sequences, and this hinders our ability to study how pleiotropy influences coding and regulatory sequence evolution. Here we use population genomics to estimate the strength of selection on coding and regulatory mutations for a transcriptional regulatory network that influences complex behavior of honey bees. We found that replacement mutations in highly connected transcription factors and target genes experience significantly stronger negative selection relative to weakly connected transcription factors and targets. Adaptively evolving proteins were significantly more likely to reside at the periphery of the regulatory network, while proteins with signs of negative selection were near the core of the network. Interestingly, connectedness and network structure had minimal influence on the strength of selection on putative regulatory sequences for both transcription factors and their targets. Our study indicates that adaptive evolution of complex behavior can arise because of positive selection on protein-coding mutations in peripheral genes, and on regulatory sequence mutations in both transcription factors and their targets throughout the network.

Keywords: Apis mellifera, network hubs, natural selection, evo devo, social evolution

\section{INTRODUCTION}

Understanding the genetics and evolution of complex traits is a central goal in biology. Behavior is a complex phenotype that exhibits a high degree of variation within an individual's lifetime, within and between populations of the same species, and between species. Behavioral genetics research conducted over the past decade has emphasized the role of conserved genes in behavioral evolution. There is good evidence that behavior, like most complex phenotypes, is controlled by gene regulatory networks that exhibit modularity and pleiotropy, and that genes and gene networks that influence behavior in one organism also influence similar behaviors in evolutionary distant species (Anholt and Mackay, 2004; Reaume and Sokolowski, 2011; Zayed and Robinson, 2012). This conservation of gene action on behavior has allowed researchers to study behavioral evolution within the framework of Evolutionary Developmental Biology (i.e., evo devo) (Carroll, 2008). The synthesis of behavioral genetics and evo devo has led to many insights (Linksvayer and Wade, 2005; Toth and Robinson, 2007, 2009), including the existence of a genetic tool kit for behavior (i.e., conserved gene modules that influence basic forms of behavior across species), and that complex behaviors can evolve through the co-option of genetic modules that control simple forms of behavior. In contrast to the evo devo paradigm, there is a burgeoning body of literature suggesting that novel taxonomically-restricted genes are important, and perhaps most prominent, in behavioral evolution (Johnson and Tsutsui, 2011; Chen et al., 2013; Ferreira et al., 2013; Simola et al., 2013; Harpur et al., 2014; Jasper et al., 2014; Sumner, 2014). Fortunately, genomics-enabled research on a variety of model and non-model organisms is providing a wealth of information on the contribution of novel and conserved genes to the genetic architecture of complex traits. Along with population genomic data on levels of selection acting on genes and regulatory sequences, evolutionary biologists are at the verge of ultimately testing the different theories of phenotypic evolution.

The different paradigms of phenotypic evolution make distinct predictions about the relative contribution of regulatory and protein-coding sequence changes. On one end of the spectrum, 
the evo devo paradigm emphasizes the role of adaptive regulatory sequence evolution (Wray, 2007; Carroll, 2008) because of the assumption that genes with multiple functions, or genes that interact with other genes, are expected to experience a great deal of constraint at their amino acid sequence (Fisher, 1930). Others have challenged this central assumption of the evo devo paradigm by arguing that seemingly "conserved" proteins, including transcription factors, have several features that allow them to "escape" the constraining influence of pleiotropy thereby allowing adaptive evolution via amino-acid changing mutations (Lynch and Wagner, 2008; Wagner and Lynch, 2008); such features include alternative splicing, modularity at the level of protein domain and structure, and the presence of mutable short or simple sequence motifs. At the other end of the spectrum, there is a growing interest in novel taxonomically restricted genes that are free to evolve new functions without suffering from the constraining effect of pleiotropy (Chen et al., 2013). Empirical evidence do not fully support any one of these three paradigms over the others-there is population genetic evidence for both adaptive protein sequence evolution and adaptive coding sequence evolution in many organisms (Andolfatto, 2005; Hoekstra and Coyne, 2007; Halligan et al., 2010, 2013; Harpur et al., 2014; Wallberg et al., 2014). However, most previous tests of these paradigms involved correlating general rates of protein evolution with molecular features of genes and their position in regulatory networks (e.g., Hahn and Kern, 2005; Kim et al., 2007; Davila-Velderrain et al., 2014); data on the actual levels of positive or negative selection on coding sequences (Assis and Kondrashov, 2014) are seldom used. Moreover, we know very virtually nothing about how pleiotropy and the structure of gene regulatory networks affect patterns of regulatory sequence evolution.

The honey bee Apis mellifera has emerged as a model organism for studying the genetics and evolution of complex behaviors (Hunt et al., 2007; Page et al., 2012; Zayed and Robinson, 2012). Here we use several powerful genomic resources developed for the honey bee to examine if regulatory networks that influence behavior follow the predictions of the evo devo paradigm for phenotypic evolution. Chandrasekaran et al. (2011) recently constructed a brain transcriptional regulatory network (TRN) influencing several aspects of worker behavior, including behavioral maturation, foraging, and colony defense. The honey bee brain TRN is highly amenable to studies of how connectedness and network topology constrain behavioral and molecular evolution, especially given the recent availability of a large population genomic dataset for the honey bee (Harpur et al., 2014), which consists of genome wide polymorphism data for 39 A. mellifera diploid genomes and genome wide divergence data between A. mellifera and its sister species A. cerana.

We used the honey bee population genomic dataset to study the strength of selection on protein and putative cis-regulatory sequences of genes in the bee brain TRN. We tested the following hypotheses from the evo devo paradigm: (1) Highly connected TFs and target genes are predicted to experience stronger negative selection on nonsynoymous mutations relative to weakly connected TFs and target genes and (2) Genes with signs of adaptive amino acid sequence evolution are expected to be less central within the regulatory network. The evo devo paradigm does not explicitly make predictions about the relationship between pleiotropy and regulatory sequence evolution, but rather predicts that the evolution of regulatory sequences should be less constrained relative to protein sequence evolution, and that regulatory mutations are more likely to fuel adaptive evolution. We compared the average selection coefficient on mutations in putative cis-regulatory regions of strongly and weakly connected genes within the TRN to explore how network properties influence regulatory sequence evolution. Our study provides an important glimpse into the evolution of regulatory networks that influence complex behaviors.

\section{MATERIALS AND METHODS SEQUENCING, ALIGNMENT, SNP CALLING AND MODIFIED McDONALD-KREITMAN (MK) TESTS}

We recently sequenced 40 honey bee genomes, each at approximately 40X coverage, using Illumina Hi-Seq technology (Harpur et al., 2014). Alignment and polymorphism identification were described in detail by Harpur et al. (2014). We used a Bayesian implementation of the McDonald-Kreitman (MK) test, using SnIPRE (Eilertson et al., 2012), to determine the population size scale selection coefficient $\gamma$ for 12,303 genes in the honey bee genome. Here, we used the population genomics dataset to study selection acting on putative cis-regulatory regions of the honey bee genome. We first estimated the number of polymorphic mutations in A. mellifera, and the number of fixed mutations between $A$. mellifera and its sister species A. cerana, in putative cis-regulatory regions of honey bee genes. Because the regulatory sequences of the honey bee genome have not been characterized, we considered the 1000 bp sequence upstream of each gene's start codon as a putative cis-regulatory region (Davidson, 2006; Li et al., 2006; Myers, 2014). We excluded upstream sequences that overlapped genes encoded by the complementary DNA strand, resulting in putative cis-regulatory regions with an average size of $905 \mathrm{bp}$. These regions are expected to contain most of the sequences important for transcriptional and translational control, including the 5'UTR and important transcription factor binding sites (Davidson, 2006; Li et al., 2006; Myers, 2014). Our cut-off would have certainly excluded some regulatory sequences that reside far upstream of genes (Negre et al., 2011)_-sequences that are currently very difficult to annotate in the honey bee. Despite this important caveat, our population genomic analyses (see results) show an overall signature of negative purifying selection within $1 \mathrm{~Kb}$ upstream of genes, which is consistent with such regions having a functional role related to gene regulation (Dunham et al., 2012; Wittkopp and Kalay, 2012). Following, Torgerson et al. (2009), we studied the evolution of cis-regulatory regions using a modified $\mathrm{MK}$ test by comparing the ratio of fixed:polymorphic mutations in a cis-regulatory sequence of a gene to same ratio for silent sites in the same gene. The modified MK test was implemented using SnIPRE (Eilertson et al., 2012), which allowed us to estimate the average population size scaled selection coefficients on regulatory sequence mutations. Similar to Harpur et al. (2014), we only used polymorphism data from African honey bee genomes, which represent a large population that is minimally impacted by human management (Harpur et al., 2012; Kent et al., 2012). 


\section{TRN CONSTRUCTION AND ANALYSIS}

The honey bee brain TRN (Chandrasekaran et al., 2011) is freely available online (Web: http://price.systemsbiology.net/ honeybee-transcriptional-regulatory-network). The dataset consisted of microarray probes for TFs and their targets in the bee brain TRN. We remapped the array probes to the honey bee's official gene set OGS v3.2 (Elsik et al., 2014) using Blastn v. 2.2.28+. We only retained probes that had perfect matches to OGS v3.2 gene predictions. We were able to blast match microarray probes to 191 transcription factors and 1597 target genes. We restricted our analyses to 184 TFs and 1521 target genes that had $\gamma$ estimates for coding and putative regulatory sequences. We estimated the number of target genes for every transcription factor ( $\mathrm{k}$ ranged from 1 to 161 ), and the number of transcription factors regulating every target ( $\mathrm{k}$ ranges from 1 to 15 ). We plotted the regulatory network using Gephi (Bastian et al., 2009) and produced a directed graph with 1504 nodes and 5149 edges representing transcription factor-target interactions. Gephi was used to estimate betweenness centrality of the genes in the network. We used the $\mathrm{R}$ package poweRlaw (Gillespie, 2014) to fit a power law distribution to TRN connectedness using established methods (Clauset et al., 2009). Statistical tests were carried out using R. We used a one-tailed test to compare the $\gamma$ of hub and non-hub TFs and targets, given a priori theoretical expectations and empirical findings regarding the relationship between pleiotropy/connectedness and molecular evolution. All other $p$-values are two-tailed. It is important to note that the honey bee brain TRN was developed by first selecting honey bee TFs that had robust orthologs to Drosophila TFs (Chandrasekaran et al., 2011); the bee brain TRN is thereby enriched for old taxonomically-conserved TFs and target genes. Our study of the bee brain TRN can therefore illuminate how ancestral gene networks influencing behaviors evolve, but tell us little about the role of taxonomically-restricted genes in behavioral evolution-a topic that we recently discussed elsewhere (Harpur et al., 2014).

\section{RESULTS \\ SELECTION ON REGULATORY AND CODING SEQUENCES IN THE HONEY BEE GENOME}

We had previously estimated the average population size scaled selection coefficient $\gamma$ on nonsynonymous mutations in 12,303 genes in the honey bee genome since divergence between A. mellifera and A. cerana (ca. 5 MYA) (Harpur et al., 2014). Here we used a variant of the MK test (Torgerson et al., 2009; implemented using Eilertson et al., 2012) to estimate the average $\gamma$ on mutations in putative cis-regulatory sequences by comparing the ratio of polymorphic:fixed mutations within $1 \mathrm{~kb}$ upstream of a gene's start codon to the ratio of polymorphic:fixed synonymous mutations at the same gene. We were able to estimate $\gamma$ on the putative cis-regulatory sequences of 10,807 genes in the honey bee genome (Figure 1). We found most (93\%) cis-regulatory sequences to have estimates of $\gamma$ consistent with neutral or nearly neutral evolution $(-1<\gamma<1)$. About $6 \%$ of cis-regulatory sequences have $\gamma<-1$, indicative of negative purifying selection, while $1 \%$ of sequences have signs of positive selection $(\gamma>1)$. In contrast to evolution of protein coding sequences (average $\gamma \sim$ 0 ), the average mutation in cis-regulatory regions appear to be

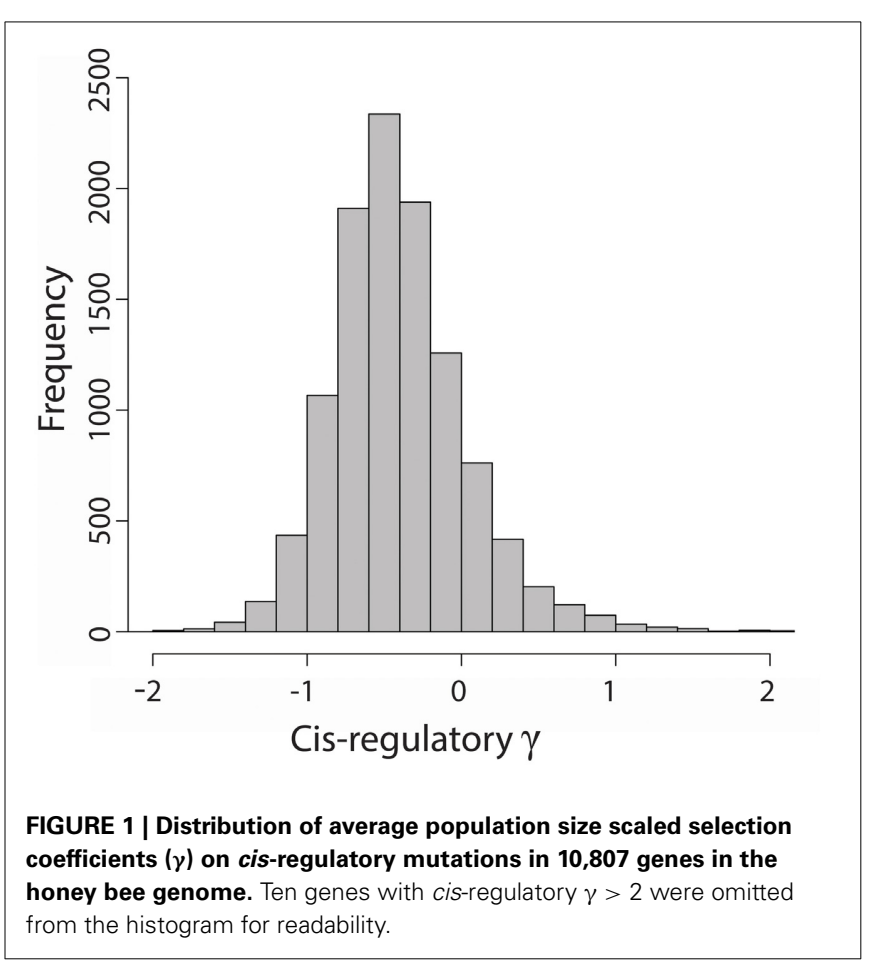

weakly deleterious (average $\gamma=-0.4$ ). This pattern was previously observed in humans (Torgerson et al., 2009) and most likely results from an observational bias: sequences from rapidly evolving regulatory regions will have many mismatches between A. mellifera and A. cerana, which results in lower alignment scores and coverage, and would have been removed from the dataset based on our quality control filters. As such, direct comparisons of the selection coefficient on coding and regulatory mutations are not appropriate. Instead, we examined the influence of a gene's connectedness and position within the TRN on regulatory and protein sequence evolution in separate analyses.

\section{NETWORK TOPOLOGY AND EVOLUTION OF TFS AND THEIR TARGET GENES}

We studied patterns of selection on coding and regulatory mutations in 170 transcription factors (TFs) and 1334 of their target genes in the honey bee brain TRN. Similar to other regulatory networks (Babu et al., 2004; Nicolau and Schoenauer, 2009), the honey bee brain TRN is approximately scale-free, whereby the distribution of connectedness $(k)$ between the network nodes (i.e., genes) has a very long tail (Supplementary Information Figure S1). The bee brain TRN contained a large number of genes with a small number of connections, and a small number of genes with a large number of connections-often called "hub" genes. The number of connections, $k$, between nodes in a scale-free network follows a power law, at least above a certain value of $k$ (Nicolau and Schoenauer, 2009). Connectedness varied between 1 and 161 in the honey bee brain TRN, and we found the tail of the connectedness distribution to follow a power law $\left(x_{\min }=42, \propto=3.00 ; H_{0}=\right.$ power law: Goodness of fit: 0.088, $p=0.32$ ). We elected to analyse the dataset by categorizing genes 
as hub or non-hub, following Wang et al. (2010a), because analyses based on linear models or correlations do not adequately deal with the properties of regulatory networks (i.e., the distribution of connections within the TRN is not normal). Following Wang et al. (2010a), we considered the top $20 \%$ of most connected TFs as hubs ( $k>44$ connections). Hub TFs were more central in the network as evidenced by a significantly higher estimate of eigenvector centrality relative to non-hub TFs (Wilcoxon test, $p<2.2 \mathrm{e}-16$ ). We found that hub TFs had a significantly lower mean coding $\gamma$ than non-hub transcription factors (Figure 2A, Wilcoxon 1-tailed $p=0.0025$ ), and that hub TFs were significantly enriched for genes with negative coding $\gamma$ (Chi square enrichment $p=0.015$ ) relative to non-hub TFs. In contrast to coding $\gamma$, hub TFs and non-hub TFs did not significantly differ with respect to cis-regulatory $\gamma$ (Figure 2C, Wilcoxon 1-tailed $p=0.27$ ). Hub and non-hub TFs did not significantly differ in terms of sequence coverage and length at regulatory and coding sites (Supplementary Information Table 1).

Similar to TFs, we used connectedness to classify target genes in the TRN into hubs (top 20\%) and non-hubs based on $k$. Hub target genes within the TRN were regulated by four or more TFs, and were significantly more central within the network relative to non-hub target genes (Wilcoxon $p=2.2 \mathrm{e}-16$ ). Similar to the
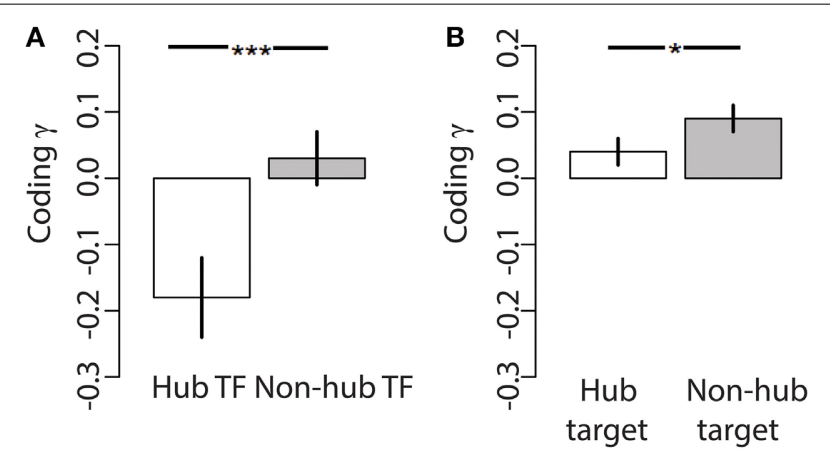

C

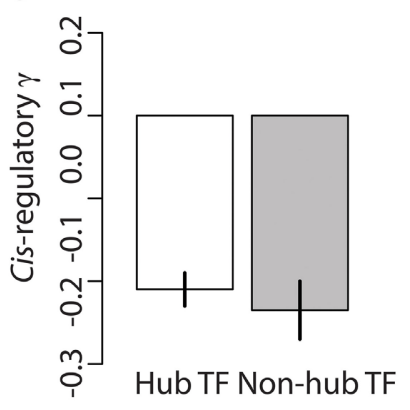

D

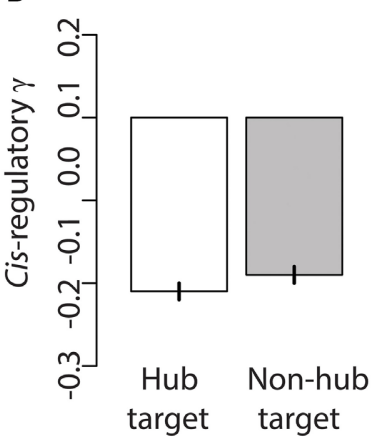

FIGURE 2 | Connectedness reduces the seletion coefficient on coding but not regulatory mutations across the honey bee TRN. Both (A) hub TFs and (B) hub target genes have signficantly stronger negative selection on their coding sequences (i.e., lower coding $\gamma$ ) relative to non-hub TFs and non-hub targets, respectively. The selection coefficient on putative cis-regulatory sequences of (C) hub TFs and (D) hub target genes do significantly differ relative to non-hub TFs and non-hub targets, respectively. Bars indicate Mean \pm SEM, ${ }^{*} p<0.05,{ }^{* * *} p<0.001$. differences between hub TFs and non-hub TFs, hub target genes had significantly lower coding $\gamma$ (Figure 2B, Wilcoxon 1-tailed $p=0.0425$ ), but not cis-regulatory $\gamma$ (Figure 2D, Wilcoxon 1tailed $p=0.12$ ) relative to non-hub target genes. Hub and non-hub target genes did not significantly differ in terms of sequence coverage and length at regulatory and coding sites (Supplementary Information Table 1).

\section{WHERE IS POSITIVE SELECTION ACTING WITHIN THE TRN?}

We mapped all genes with signatures of positive selection on coding and cis-regulatory sequences in the TRN (Figure 3). We also estimated betweenness for each gene in the TRN; betweenness is a global measure of centrality (Borgatti and Everett, 2006) which ranges from 1 , indicating most central or at the core of the network, to 0 , indicating the outside perimeter or the periphery of the network. We compared the average betweenness of genes with substantial signs of positive $(\gamma>1)$ and negative $(\gamma<-1)$ selection. We found that proteins with signatures of positive selection on their coding sequences had significantly lower betweenness relative to proteins with signatures of negative selection, indicating that adaptively evolving proteins are often more distant from the network core relative to proteins with signs of negative selection (Figure 4A, Wilcoxon, two tailed $p=0.04$ ). In contrast, we did not find a significant difference in the betweenness of genes with positive selection on their cis-regulatory sequences relative to those with negative selection on their cis-regulatory sequences (Figure 4B, Wilcoxon two-tailed $p=0.4$ ). This indicates that genes with regulatory sequences experiencing positive selection reside in approximately the same locations within the TRN as genes with regulatory sequences experiencing negative selection.

\section{DISCUSSION}

We examined how gene position within a network influenced the average selection coefficient $\gamma$ on putative cis-regulatory and replacement mutations in 1504 genes in the honey bee brain TRN. Our results support a "mosaic" view of phenotypic evolution by illuminating how the scale-free properties of regulatory networks (Wang et al., 2010b; Le Nagard et al., 2011; Wagner and Zhang, 2011) facilitate adaptive evolution involving both coding and regulatory mutations.

Several lines of evidence suggest that the most connected, and likely most pleiotropic, proteins within the bee brain TRN experience the greatest levels of purifying selection, as predicted by Fisher (1930) and the Evo Devo paradigm (Carroll, 2008). Despite the large number of factors that influence the rate of molecular evolution of genes (Xia et al., 2009) we consistently found that the most connected genes in the TRN had the strongest signatures of negative selection on their coding sequence. In brief, transcription factors that regulate hundreds of target genes experience, on average, stronger negative selection on their coding sequence relative to transcription factors the regulate a few target genes (Figure 2A). Hub transcription factors likely have to interact with many other co-factors, in addition to binding target promoter sites, which may be responsible for the stronger levels of purifying selection on their amino acid sequence. Similar to hub transcription factors, hub target genes that are regulated by 

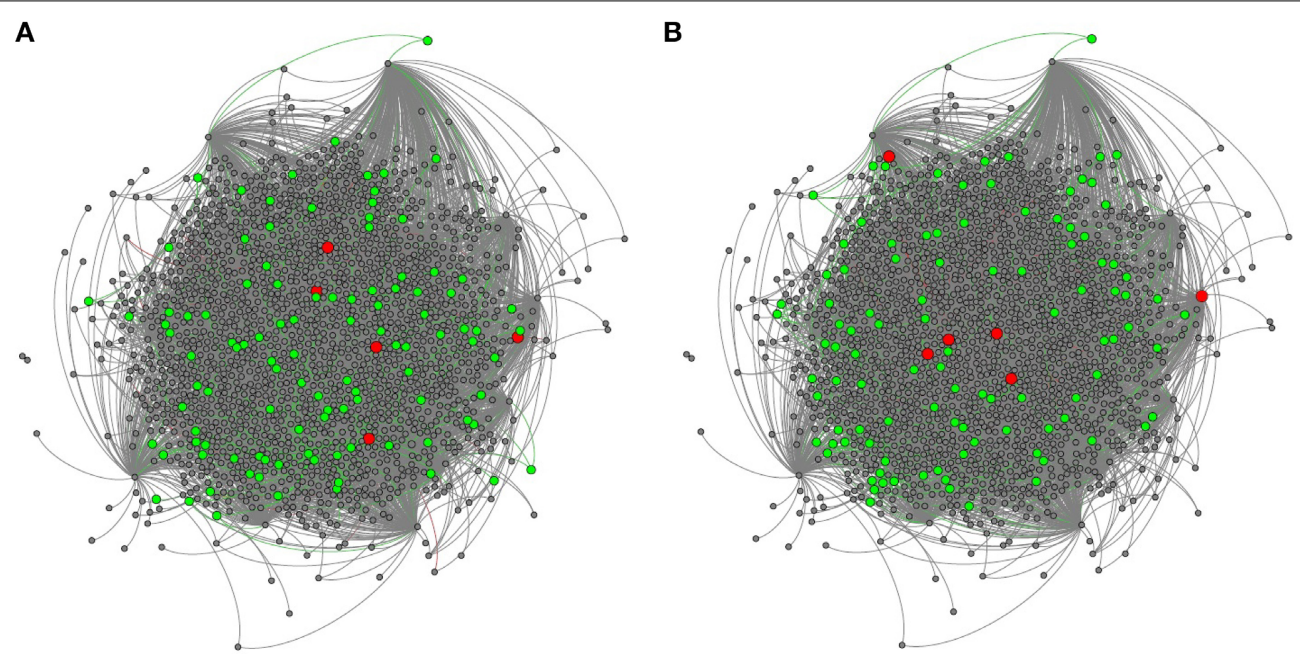

FIGURE 3 | The honey bee brain TRN highlighting genes with adaptively evolving (A) cis-regulatory and (B) coding sequences. Adaptively evolving transcription factors are highlighted in red, while adaptively evolving targets are highlighted in green.

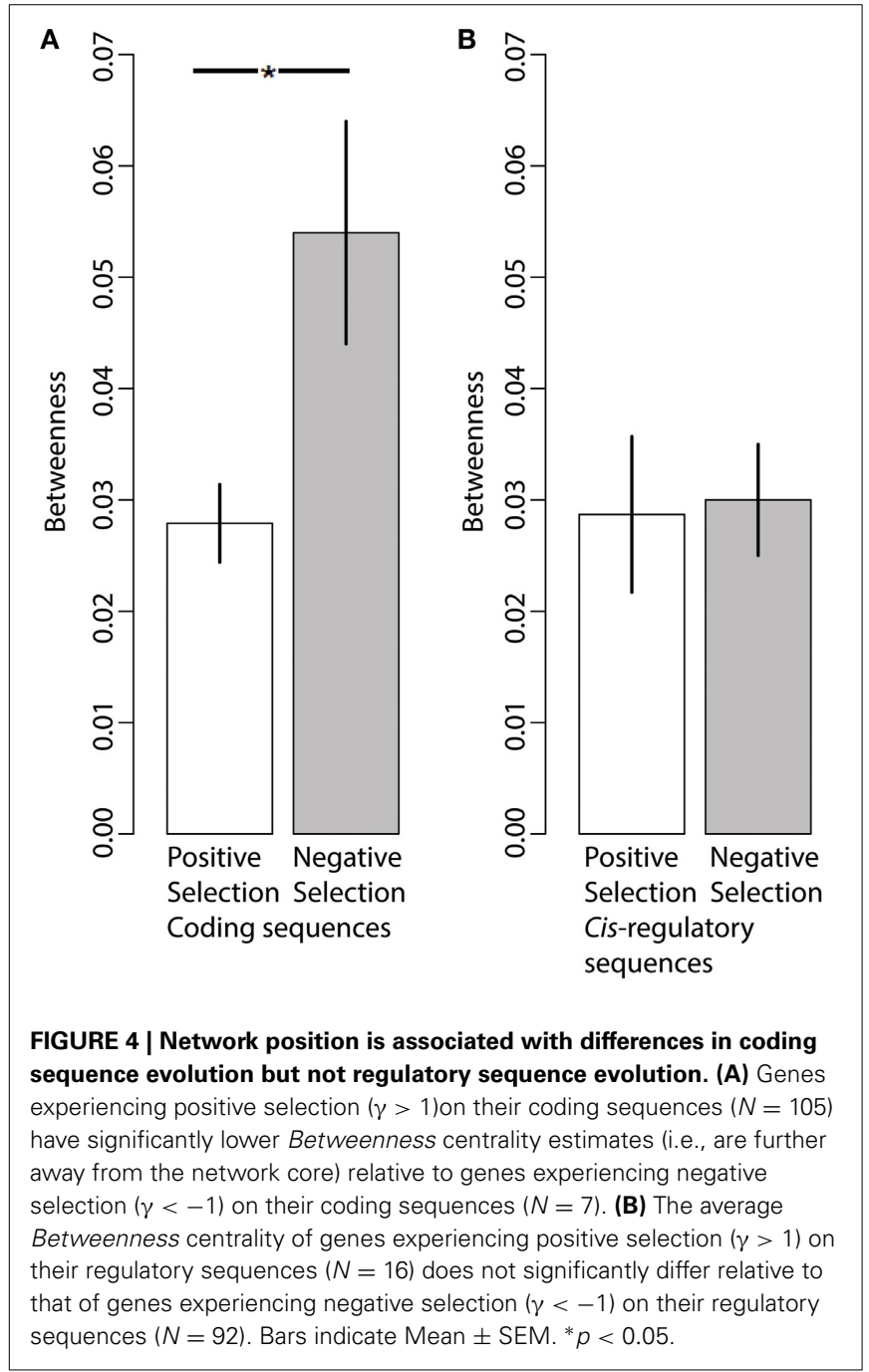

many transcription factors experience stronger negative selection on their coding sequence relative to target genes that are regulated by a few transcription factors. Target genes that are regulated by multiple TFs may be expressed in multiple tissues or during multiple contexts relative to target genes regulated by a few TFs, resulting in greater pleiotropy and stronger purifying selection, as evident from our analysis (Figure 2B). It is important to note that several genes within the TRN had signs of adaptive protein evolution; most of these genes were transcription factor targets, and most resided near the periphery of the TRN. Lynch and Wagner (2008) and Wagner and Lynch (2008) previously argued that proteins, including conserved TFs, have features that allow them to escape from the negative effects of pleiotropy. Our population genomic data are not fully consistent with the Lynch and Wagner hypotheses because the most central and most connected TFs or targets do experience stronger levels of negative selection vs. peripheral and weakly connected TFs or targets; a relationship that is more inline with the classic evo devo paradigm. We strongly believe that the structure of TRNs hold the key for reconciling the predictions of the evo devo paradigm with the empirical data showing that amino-acid changes do contribute to adaptive evolution. The classic evo devo paradigm assumes that most genes are constrained by pleiotropy, while studies of TRN structure clearly show that only a few genes are highly connected and central, while most genes are weakly connected and peripheral. Although pleiotropy does appear to curtail adaptive protein sequence evolution of the few most connected and most central genes within a TRN, adaptive protein evolution is still a powerful evolutionary force for most TRN genes that reside at the network periphery.

In stark contrast to the influence of TRN topology on protein coding evolution, we found that connectedness matters little with respect to levels of selection on putative cisregulatory regions. The average selection coefficient on regulatory sequence mutations of hub transcription factors was similar to 
that of non-hub transcription factors (Figure 2C). Similarly, the selection coefficient on regulatory sequences of hub target genes was similar to those of non-hub target genes. Genes with signs of adaptive regulatory sequence evolution were found in similar locations within the TRN as genes with negative selection on their regulatory sequences. Our analysis indicates that network properties do not significantly shape the selection pressures acting on regulatory sequences within the TRN. It is not clear how this evidence support the evo devo paradigm because the evo devo paradigm does not make explicit predictions about the relationship between pleiotropy, connectedness and regulatory sequence evolution. On one hand, our finding that putative cisregulatory sequences evolve independently of TRN connectedness and topology appears to support an important assumption of the evo devo paradigm: pleiotropy or connectedness of a protein only influences the protein's amino acid sequence, not its cis-regulatory sequence. On the other hand, another interpretation of the evo devo paradigm suggests that the most connected and pleiotropic genes should have the greatest levels of adaptive regulatory evolution, while the least connected genes should have the least levels of adaptive regulatory evolution (i.e., regulatory sequence evolution compensates for constrained amino acid sequences); our findings do not support this idea. It would appear that adaptive regulatory sequence evolution can occur throughout any compartment of the regulatory network.

Our analyses shed light on the evolution of regulatory networks influencing complex behavior. Highly connected genes within the honey bee brain TRN exhibit stronger patterns of purifying selection on amino acid replacement mutations similar to highly connected genes in other types of networks studied so far. Also, genes with signs of adaptive protein evolution tend to be concentrated at the network periphery, as previously documented for proteins in the Human Interactome (Kim et al., 2007). We found that connectedness does not influence the strength of selection on regulatory sequences of genes in the bee brain TRN. Our study suggests that the properties of regulatory networks, with a few large modules and many small modules, allows for both coding and regulatory sequence mutations to contribute to adaptive evolution. Based on our findings, we expect adaptive evolution of regulatory networks influencing complex traits to proceed through positive selection on coding mutations in peripheral genes and on regulatory mutations in TFs and their targets across the regulatory network. We had previously presented strong evidence that novel taxonomically-restricted genes have the highest rates of adaptive protein evolution in the honey bee genome (Harpur et al., 2014). A recent analysis also pointed to an increased expansion of regulatory sequences in social genomes (Simola et al., 2013). Going forward, it will be important to study how novel taxonomically restricted genes interact with conserved TRN modules with expanded regulatory features to influence the evolution of complex behaviors in social insects.

\section{ACKNOWLEDGMENTS}

We thank K. Eilertson for help with SnIPRE, the N. Price and G. Robinson groups for constructing the honey bee brain TRN and for making this valuable dataset publically available, and two anonymous referees for providing insightful comments on our mansucirpt. This study was supported by a Discovery Grant from the Natural Sciences and Engineering Research Council of Canada and an Early Researcher Award from the Ontario Ministry of Research and Innovation (to Amro Zayed). Brock A. Harpur was supported by an Elia Doctoral Scholarship from York University.

\section{SUPPLEMENTARY MATERIAL}

The Supplementary Material for this article can be found online at: http://www.frontiersin.org/journal/10.3389/fgene. 2014.00431/abstract

\section{Figure S1 | Degree distribution of connectedness in the TRN.}

\section{REFERENCES}

Andolfatto, P. (2005). Adaptive evolution of non-coding DNA in Drosophila. Nature 437, 1149-1152. doi: 10.1038/nature04107

Anholt, R. R. H., and Mackay, T. F. C. (2004). Quantitative genetic analyses of complex behaviours in Drosophila. Nat. Rev. Genet. 5, 838-849. doi: $10.1038 / \operatorname{nrg} 1472$

Assis, R., and Kondrashov, A. S. (2014). Conserved proteins are fragile. Mol. Biol. Evol. 31, 419-424. doi: 10.1093/molbev/mst217

Babu, M. M., Luscombe, N. M., Aravind, L., Gerstein, M., and Teichmann, S. A. (2004). Structure and evolution of transcriptional regulatory networks. Curr. Opin. Struct. Biol. 14, 283-291. doi: 10.1016/j.sbi.2004.05.004

Bastian, M., Heymann, J., and Jacomy, M. (2009). "Gephi: an open source software for exploring and manipulating networks," in International AAAI Conference on Weblogs and Social Media (Paris).

Borgatti, S. P., and Everett, M. G. (2006). A graph-theoretic perspective on centrality. Soc. Netw. 28, 466-484. doi: 10.1016/j.socnet.2005.11.005

Carroll, S. B. (2008). Evo-devo and an expanding evolutionary synthesis: a genetic theory of morphological evolution. Cell 134, 25-36. doi: 10.1016/j.cell.2008.06.030

Chandrasekaran, S., Ament, S. A., Eddy, J. A., Rodriguez-Zas, S. L., Schatz, B. R., Price, N. D., et al. (2011). Behavior-specific changes in transcriptional modules lead to distinct and predictable neurogenomic states. Proc. Natl. Acad. Sci. U.S.A. 108, 18020-18025. doi: 10.1073/pnas.1114093108

Chen, S. D., Krinsky, B. H., and Long, M. Y. (2013). New genes as drivers of phenotypic evolution. Nat. Rev. Genet. 14, 645-660. doi: 10.1038/ nrg3521

Clauset, A., Shalizi, C. R., and Newman, M. E. J. (2009). Power-law distributions in empirical data. Siam Rev. 51, 661-703. doi: 10.1137/070710111

Davidson, E. H. (2006). The Regulatory Genome: Gene Regulatory Networks in Development and Evolution. Burlington, VT; San Diego, CA: Academic.

Davila-Velderrain, J., Servin-Marquez, A., and Alvarez-Buylla, E. R. (2014). Molecular evolution constraints in the floral organ specification gene regulatory network module across 18 angiosperm genomes. Mol. Biol. Evol. 31, 560-573. doi: $10.1093 / \mathrm{molbev} / \mathrm{mst} 223$

Dunham, I., Kundaje, A., Aldred, S. F., Collins, P. J., Davis, C., Doyle, F., et al. (2012). An integrated encyclopedia of DNA elements in the human genome. Nature 489, 57-74. doi: 10.1038/nature11247

Eilertson, K. E., Booth, J. G., and Bustamante, C. D. (2012). SnIPRE: selection inference using a poisson random effects model. PLoS Comput. Biol. 8:E1002806. doi: 10.1371/journal.pcbi.1002806

Elsik, C. G., Worley, K. C., Bennett, A. K., Beye, M., Camara, F., Childers, C. P., et al. (2014). Finding the missing honey bee genes: lessons learned from a genome upgrade. BMC Genomics 15:86. doi: 10.1186/1471-2164-15-86

Ferreira, P. G., Patalano, S., Chauhan, R., Ffrench-Constant, R., Gabaldon, T., Guigo, R., et al. (2013). Transcriptome analyses of primitively eusocial wasps reveal novel insights into the evolution of sociality and the origin of alternative phenotypes. Genome Biol. 14:R20. doi: 10.1186/gb-201314-2-r20

Fisher, R. A. (1930). The Genetic Theory of Natural Selection. New York, NY: Dover. Gillespie, C. S. (2014). Fitting heavy tailed distributions: the powerlaw package. $R$ package version 0.20 .25 (Newcastle, UK). 
Hahn, M. W., and Kern, A. D. (2005). Comparative genomics of centrality and essentiality in three eukaryotic protein-interaction networks. Mol. Biol. Evol. 22, 803-806. doi: 10.1093/molbev/msi072

Halligan, D. L., Kousathanas, A., Ness, R. W., Harr, B., Eory, L., Keane, T. M., et al. (2013). Contributions of protein-coding and regulatory change to adaptive molecular evolution in murid rodents. PLoS Genet. 9:e1003995. doi: 10.1371/journal.pgen.1003995

Halligan, D. L., Oliver, F., Eyre-Walker, A., Harr, B., and Keightley, P. D. (2010). Evidence for pervasive adaptive protein evolution in wild mice. PLoS Genet. 6:e1000825. doi: 10.1371/journal.pgen.1000825

Harpur, B. A., Kent, C. F., Molodtsova, D., Lebon, J. M., Alqarni, A. S., Owayss, A. A., et al. (2014). Population genomics of the honey bee reveals strong signatures of positive selection on worker traits. Proc. Natl. Acad. Sci. U.S.A. 111, 2614-2619. doi: 10.1073/pnas.1315506111

Harpur, B. A., Minaei, S., Kent, C. F., and Zayed, A. (2012). Management increases genetic diversity of honey bees via admixture. Mol. Ecol. 21, 4414-4421. doi: 10.1111/j.1365-294X.2012.05614.x

Hoekstra, H. E., and Coyne, J. A. (2007). The locus of evolution: evo devo and the genetics of adaptation. Evolution 61, 995-1016. doi: 10.1111/j.15585646.2007.00105.x

Hunt, G. J., Amdam, G. V., Schlipalius, D., Emore, C., Sardesai, N., Williams, C. E., et al. (2007). Behavioral genomics of honeybee foraging and nest defense. Naturwissenschaften 94, 247-267. doi: 10.1007/s00114-006-0183-1

Jasper, W. C., Linksvayer, T. A., Atallah, J., Friedman, D., Chiu, J. C., and Johnson, B. R. (2014). Large scale coding sequence change underlies the evolution of post-developmental novelty in honey bees. Mol. Biol. Evol. doi: 10.1093/molbev/msu292

Johnson, B. R., and Tsutsui, N. D. (2011). Taxonomically restricted genes are associated with the evolution of sociality in the honey bee. BMC Genomics 12:164. doi: 10.1186/1471-2164-12-164

Kent, C. F., Minaei, S., Harpur, B. A., and Zayed, A. (2012). Recombination is associated with the evolution of genome structure and worker behavior in honey bees. Proc. Natl. Acad. Sci. U.S.A. 109, 18012-18017. doi: 10.1073/pnas.1208094109

Kim, P. M., Korbel, J. O., and Gerstein, M. B. (2007). Positive selection at the protein network periphery: evaluation in terms of structural constraints and cellular context. Proc. Natl. Acad. Sci. U.S.A. 104, 20274-20279. doi: 10.1073/pnas.0710183104

Le Nagard, H., Chao, L., and Tenaillon, O. (2011). The emergence of complexity and restricted pleiotropy in adapting networks. BMC Evol. Biol. 11:326. doi: 10.1186/1471-2148-11-326

Li, Y., Lee, K. K., Walsh, S., Smith, C., Hadingham, S., Sorefan, K., et al. (2006). Establishing glucose- and ABA-regulated transcription networks in Arabidopsis by microarray analysis and promoter classification using a Relevance Vector Machine. Genome Res. 16, 414-427. doi: 10.1101/gr.4237406

Linksvayer, T. A., and Wade, M. J. (2005). The evolutionary origin and elaboration of sociality in the aculeate Hymenoptera: maternal effects, sib-social effects, and heterochrony. Q. Rev. Biol. 80, 317-336. doi: 10.1086/432266

Lynch, V. J., and Wagner, G. P. (2008). Resurrecting the role of transcription factor change in developmental evolution. Evolution 62, 2131-2154. doi: 10.1111/j.1558-5646.2008.00440.x

Myers, A. J. (2014). "The genetics of gene expression: multiple layers and muliple players," in The OMiCs: Applications in Neuroscience, ed G. Coppola (Oxford: Oxford University Press), 132-154.

Negre, N., Brown, C. D., Ma, L. J., Bristow, C. A., Miller, S. W., Wagner, U., et al. (2011). A cis-regulatory map of the Drosophila genome. Nature 471, 527-531. doi: 10.1038/nature09990

Nicolau, M., and Schoenauer, M. (2009). On the evolution of scale-free topologies with a gene regulatory network model. Biosystems 98, 137-148. doi: 10.1016/j.biosystems.2009.06.006

Page, R. E., Rueppell, O., and Amdam, G. V. (2012). Genetics of reproduction and regulation of honeybee (Apis mellifera L.) social behavior. Annu. Rev. Genet. 46, 97-119. doi: 10.1146/annurev-genet-110711-155610

Reaume, C. J., and Sokolowski, M. B. (2011). Conservation of gene function in behaviour. Philos. Trans. R. Soc. B Biol. Sci. 366, 2100-2110. doi: $10.1098 /$ rstb. 2011.0028
Simola, D. F., Wissler, L., Donahue, G., Waterhouse, R. M., Helmkampf, M., Roux, J., et al. (2013). Social insect genomes exhibit dramatic evolution in gene composition and regulation while preserving regulatory features linked to sociality. Genome Res. 23, 1235-1247. doi: 10.1101/gr. 155408.113

Sumner, S. (2014). The importance of genomic novelty in social evolution. Mol. Ecol. 23, 26-28. doi: 10.1111/mec. 12580

Torgerson, D. G., Boyko, A. R., Hernandez, R. D., Indap, A., Hu, X., White, T. J., et al. (2009). Evolutionary processes acting on candidate cis-regulatory regions in humans inferred from patterns of polymorphism and divergence. PLoS Genet. 5:e1000592. doi: 10.1371/journal.pgen.1000592

Toth, A. L., and Robinson, G. E. (2007). Evo-devo and the evolution of social behavior. Trends Genet. 23, 334-341. doi: 10.1016/j.tig.2007. 05.001

Toth, A. L., and Robinson, G. E. (2009). Evo-devo and the evolution of social behavior: brain gene expression analyses in social insects. Cold Spring Harb. Symp. Quant. Biol. 74, 419-426. doi: 10.1101/sqb.2009.74.026

Wagner, G. P., and Lynch, V. J. (2008). The gene regulatory logic of transcription factor evolution. Trends Ecol. Evol. 23, 377-385. doi: 10.1016/j.tree.2008. 03.006

Wagner, G. P., and Zhang, J. (2011). The pleiotropic structure of the genotypephenotype map: the evolvability of complex organisms. Nat. Rev. Genet. 12, 204-213. doi: 10.1038/nrg2949

Wallberg, A., Han, F., Wellhagen, G., Dahle, B., Kawata, M., Haddad, N., et al. (2014). A worldwide survey of genome sequence variation provides insight into the evolutionary history of the honeybee Apis mellifera. Nat Genet. Available Online at: http://www.nature.com/ng/journal/vaop/ncurrent/abs/ng. 3077.html - supplementary-information.

Wang, Y., Franzosa, E. A., Zhang, X. S., and Xia, Y. (2010a). Protein evolution in yeast transcription factor subnetworks. Nucleic Acids Res. 38, 5959-5969. doi: 10.1093/nar/gkq353

Wang, Z., Liao, B. Y., and Zhang, J. (2010b). Genomic patterns of pleiotropy and the evolution of complexity. Proc. Natl. Acad. Sci. U.S.A. 107, 18034-18039. doi: 10.1073/pnas.1004666107

Wittkopp, P. J., and Kalay, G. (2012). Cis-regulatory elements: molecular mechanisms and evolutionary processes underlying divergence. Nat. Rev. Genet. 13, 59-69. doi: 10.1038/nrg3095

Wray, G. A. (2007). The evolutionary significance of cis-regulatory mutations. Nat. Rev. Genet. 8, 206-216. doi: 10.1038/nrg2063

Xia, Y., Franzosa, E. A., and Gerstein, M. B. (2009). Integrated assessment of genomic correlates of protein evolutionary rate. PLoS Comput. Biol. 5:e1000413. doi: 10.1371/journal.pcbi.1000413

Zayed, A., and Robinson, G. E. (2012). Understanding the relationship between brain gene expression and social behavior: lessons from the honey bee. Annu. Rev. Genet. 46, 591-615. doi: 10.1146/annurev-genet-110711155517

Conflict of Interest Statement: The authors declare that the research was conducted in the absence of any commercial or financial relationships that could be construed as a potential conflict of interest.

Received: 03 October 2014; accepted: 21 November 2014; published online: 23 December 2014.

Citation: Molodtsova D, Harpur BA, Kent CF, Seevananthan K and Zayed A (2014) Pleiotropy constrains the evolution of protein but not regulatory sequences in a transcription regulatory network influencing complex social behaviors. Front. Genet. 5:431. doi: $10.3389 /$ fgene.2014.00431

This article was submitted to Evolutionary and Population Genetics, a section of the journal Frontiers in Genetics.

Copyright (C) 2014 Molodtsova, Harpur, Kent, Seevananthan and Zayed. This is an open-access article distributed under the terms of the Creative Commons Attribution License (CC BY). The use, distribution or reproduction in other forums is permitted, provided the original author(s) or licensor are credited and that the original publication in this journal is cited, in accordance with accepted academic practice. No use, distribution or reproduction is permitted which does not comply with these terms. 